\title{
Auditory Gating in Hearing Loss
}

\author{
Julia Campbell ${ }^{1,2}$ Mashhood Nielsen ${ }^{1,2}$ Connor Bean ${ }^{1,2}$ Alison LaBrec ${ }^{1,2}$ \\ ${ }^{1}$ Department of Communication Sciences and Disorders, University \\ of Texas at Austin, Austin, Texas \\ ${ }^{2}$ Central Sensory Processes Laboratory, University of Texas at Austin, \\ Austin, Texas \\ J Am Acad Audiol 2020;31:559-565. \\ Address for correspondence Julia Campbell, PhD, AuD, CCC-A, F-AAA, \\ Julia.Campbell@Austin.UTexas.EDU.
}

\begin{abstract}
Keywords

- hearing loss

- CAEP

- auditory gating

- current density reconstruction

- sLORETA

- inhibition

Background Sensory gating is a measure used to evaluate inhibitory deficits underlying neurological disorders. However, the effects of hearing loss $(\mathrm{HL})$, thought to decrease inhibition, remain unknown on gating function.

Purpose The goal of this study was to investigate gating performance in $\mathrm{HL}$.

Research Design This was a prospective, cross-sectional study with independent group comparison and correlational design.

Study Sample Eleven adults (mean age/standard deviation $=47.546 \pm 7.967$ years) with normal hearing $(\mathrm{NH})$ and 11 adults (mean age/standard deviation $=56.273 \pm 13.871$ years) with mild-moderate high-frequency $\mathrm{HL}$.

Data Collection and Analysis Cortical auditory evoked potentials (CAEPs) were recorded in response to tonal pairs via high-density electroencephalography. The CAEP response to the second tone in the pair (S2) was compared with the response to the first tone in the pair (S1) within groups. Amplitude gating indices were compared between groups and correlated with auditory behavioral measures. Current density reconstructions were performed to estimate cortical gating generators.

Results Amplitude gating indices were decreased and correlated with elevated auditory thresholds. Gating generators in temporal, frontal, and prefrontal regions were localized in the $\mathrm{NH}$ group, while $\mathrm{HL}$ gating was localized in mainly temporal and parietal areas.

Conclusions Reduced inhibition may be associated with compensatory cortical gating networks in $\mathrm{HL}$ and should be considered when utilizing gating in clinical populations.
\end{abstract}

Sensory gating is a measure used to study degraded central inhibitory function underlying various disorders, including autism spectrum disorder, schizophrenia, and tinnitus. ${ }^{1-4}$ The role of inhibition in gating function is to filter out nonnovel input, leaving adequate resources for the brain to process relevant information. ${ }^{5}$ This process appears to be regulated by endogenous mechanisms through the action of nicotinic receptors (nAChR) on inhibitory interneurons in the hippocampus, as well as distributed nAChR activity in temporoparietal, temporofrontal, and prefrontal cortical networks. ${ }^{6-8}$ In certain disorders, these underlying inhibitory systems may be deficient, allowing for abnormal sensory perceptions to occur. ${ }^{5}$

Through auditory gating, inhibition is evaluated using cortical auditory evoked potentials (CAEPs) recorded via electroencephalography (EEG) in response to repeated pairs of identical acoustic stimuli (S1 and S2). ${ }^{5}$ Normal gating is observed through amplitude suppression of the CAEP S2 response in relation to CAEP S1 amplitude, as the second stimulus in the pair is deemed nonnovel. ${ }^{5}$ Inhibition can then be quantified through the peak amplitude ratio (P50 S2/P50 received

July 23, 2019

accepted after revision

December 30, 2019

published online

April 27, 2020
Copyright $\odot 2020$ by the American

Academy of Audiology. All rights reserved. Thieme Medical Publishers, Inc., 333 Seventh Avenue, New York, NY 10001 , USA.

Tel: +1(212) 760-0888.
DOI https://doi.org/ 10.1055/s-0040-1709517. ISSN 1050-0545. 
S1) and difference indices (P50 S1-P50 S2). P50 ratio values less than 0.500 and difference values greater than $0 \mu \mathrm{V}$ are reflective of normal suppression, ${ }^{9}$ and act as a biomarker of deficient central inhibition. ${ }^{5}$

While endogenous mechanisms play a significant role in gating processes, exogenous factors should be considered as well. For instance, peripheral auditory deafferentation, or sensorineural hearing loss (HL), has been found to adversely impact central inhibitory mechanisms through a reduction of inhibitory inputs and subsequent imbalance between excitatory and inhibitory systems. ${ }^{10-12}$ Indeed, CAEP studies in humans have described increased amplitude of peak components to correlate with measures of HL, specifically via the CAEP P2 component, which may arise from decreased neural inhibition. ${ }^{13-17}$ Thus, it may be hypothesized that HL would alter aspects of the gating response, which is composed of CAEP amplitude indices.

However, to our knowledge, the specific effects of HL on sensory gating remain unknown, as studies routinely exclude participants with auditory deficits. ${ }^{18}$ Because this measure is used to study inhibitory deficits in clinical populations, ${ }^{1,4,18}$ including recent research targeting tinnitus, ${ }^{2,3}$ it is necessary to understand the possible effects of $\mathrm{HL}$ on the gating response. Therefore, we examined gating performance in adults with normal hearing $(\mathrm{NH})$ and mild-moderate highfrequency (HF) sensorineural HL. CAEPs were recorded via high-density EEG in response to tonal pairs. Peak amplitude and latency were compared within groups, while the peak amplitude ratio and difference indices were compared between groups. It was hypothesized that decreased gating function would be observed in HL, and would correlate with auditory thresholds. The second goal of the study was to assess group differences in cortical gating networks via current density reconstructions (CDRs). We hypothesized that the HL group would present with incomplete activation of those temporal, frontal, and prefrontal gating networks that have been identified in individuals with $\mathrm{NH}^{6,7}$

\section{Methods}

\section{Participants}

This study was approved by the University of Texas Institutional Review Board. Twenty-two adults provided informed written consent and were grouped according to pure-tone thresholds above $25 \mathrm{~dB}$ HL. A requirement for study inclusion was that no participant report tinnitus or score above 0 on the Tinnitus Handicap Inventory. ${ }^{3,19}$ There was no significant age difference between $\mathrm{NH}(n=11$, mean age/standard deviation $=47.546 \pm 7.967$ years $)$ or HL groups $(n=11$, mean age/standard deviation $=56.273 \pm 13.871$ years $)[F(1$, $20)=3.274, p=0.085$ ]. Nine females and one male were included in the $\mathrm{NH}$ group, while four females and seven males made up the HL group. Seventeen participants denied smoking, while two $\mathrm{NH}$ and three HL participants did not answer this question. ${ }^{6,7}$ One $\mathrm{NH}$ participant reported an unspecified psychological diagnosis, one reported migraine, and one HL participant reported migraine.

\section{Audiometry and Speech Perception}

Audiometric thresholds were measured bilaterally at 0.250 to $8 \mathrm{kHz}$ via 3M E-A-R TONE GOLD 3A insert earphones. The criterion for HL was $25 \mathrm{~dB} \mathrm{HL}^{15}$ (-Fig. 1). Bone conduction thresholds were obtained to verify type of HL. Pure-tone averages (PTAs) were calculated at $0.500,1$, and $2 \mathrm{kHz}$, and HF PTAs at 4 and $8 \mathrm{kHz}$. No participant reported history of using an amplification device.

\section{Right Ear}

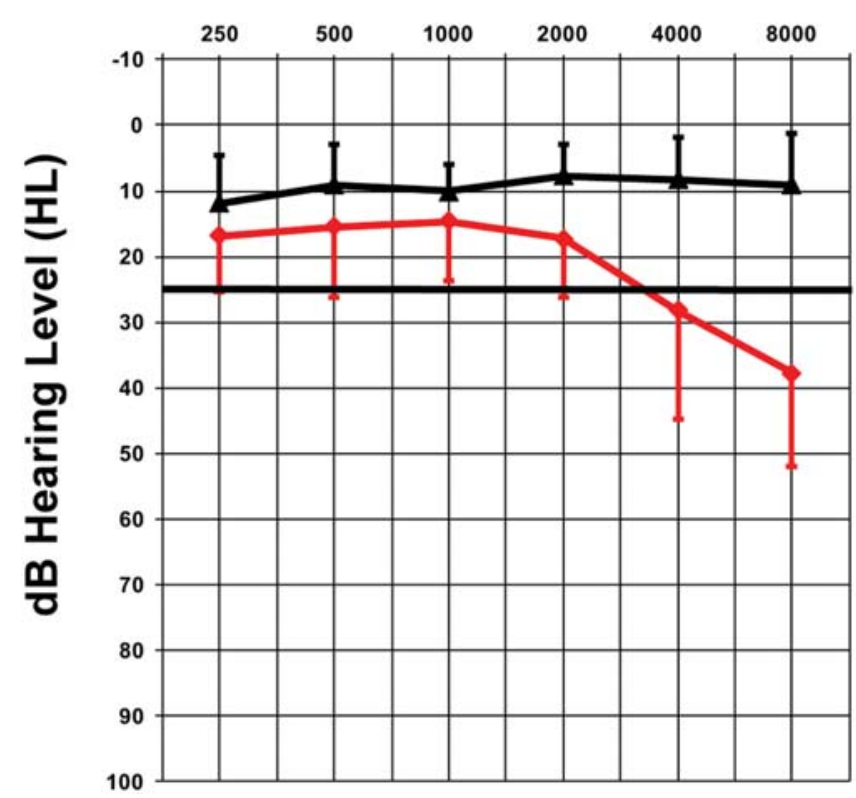

\section{Left Ear}

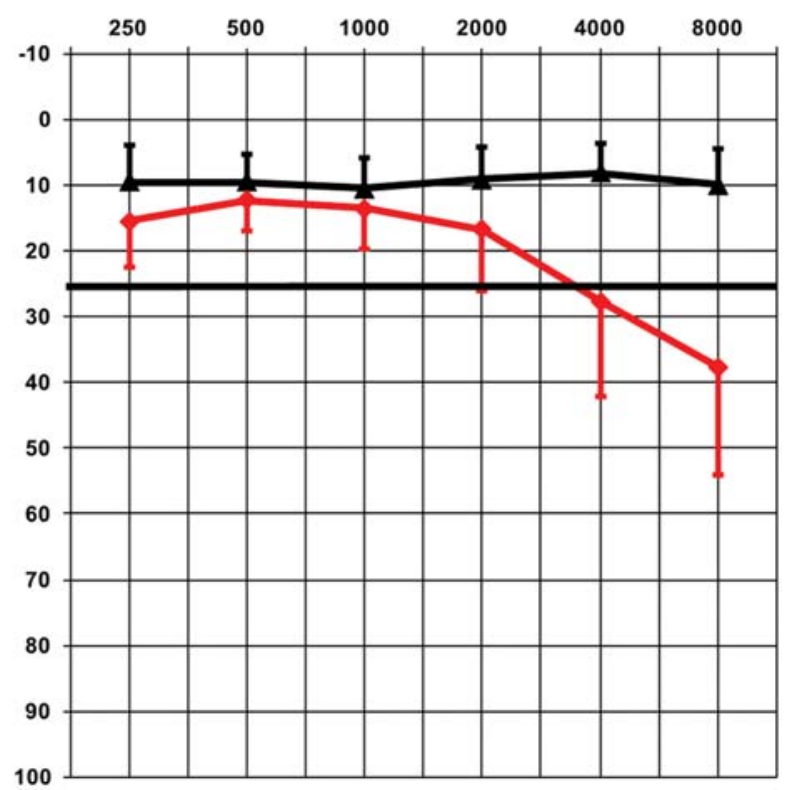

Fig. 1 Audiometric thresholds. Pure-tone thresholds for $\mathrm{NH}$ (black, $n=11$ ) and $\mathrm{HL}$ groups (red, $n=11$ ). The criterion for hearing loss was $25 \mathrm{~dB}$ $\mathrm{HL}$ (horizontal black line). Error bars represent one standard deviation. $\mathrm{HL}$, hearing loss; $\mathrm{NH}$, normal hearing. 
Signal-to-noise ratio (SNR) loss was assessed using the QuickSIN Speech-in-Noise Test (Etymotic Research) to determine a relationship between gating indices and auditory performance in a degraded condition. Recorded sentences were delivered at a level of $70 \mathrm{~dB}$ HL through a speaker placed at $0^{\circ}$ azimuth, while background noise varied from 25 to $0 \mathrm{~dB}$ SNR. Individual SNR loss was calculated from the average of two lists.

\section{Auditory Gating Paradigm}

Participants were fit with a 128-channel electrode net (Electrical Geodesics, Inc.) and underwent testing in a sound booth. The EEG sampling rate was $1 \mathrm{kHz}$, with a band-pass filter set at 0.100 to $200 \mathrm{~Hz}$. Ocular electrodes were utilized for offline rejection of myogenic activity.

Stimuli consisted of $0.25 \mathrm{kHz}$ tonal pairs, a frequency chosen to ensure audibility regardless of hearing status at higher frequencies, with a duration of 50 milliseconds and 10 milliseconds-linear rise/fall times. The interstimulus interval was 500 milliseconds and the intertrial interval was 7 seconds. Seven hundred tonal pairs were presented at a level of $50 \mathrm{~dB} \mathrm{HL}$ through two speakers placed at $\pm 45^{\circ}$ azimuth while participants watched a muted movie with subtitles. Please see Campbell et $\mathrm{al}^{2,3}$ for additional details regarding the paradigm.

\section{EEG Analyses}

A 1-Hz high-pass filtered was applied and event epochs were created with -100 milliseconds prestimulus and 350 milliseconds poststimulus periods. Data were exported to EEGLAB $^{20}$ and baseline-corrected according to the prestimulus period. Channels with amplitude greater than a standard deviation value of $\pm 3 \mu \mathrm{V}$ were deleted, followed by artifact rejection using the same criterion. Deleted channels were interpolated using a spherical interpolation algorithm. A total of 529.909 sweeps were included in the $\mathrm{NH}$ CAEP S1 average, and 525.546 sweeps in the CAEP S2 average. There were 460.546 sweeps accepted in the HL CAEP S1 average, and 465.909 sweeps in the CAEP S2 average. There was no significant difference between groups for the number of sweeps in the CAEP S1 average $[F(1,20)=3.586, p=0.073]$ or the CAEP S2 average $[F(1,20)=3.008, p=0.098]$.

Thirteen electrodes were averaged to create a frontal region of interest (3, 4, 5, 9 [Fp2], 10, 11 [Fz], 12, 15, 16, 18, 19, 22 [Fp1], 23). 2,3 Baseline to peak amplitude and latency of the CAEP response components were compared statistically within each group and marked as follows: P50 45-85 milliseconds, N1 90-140 milliseconds, and P2 140-220 milliseconds. Peak amplitude ratio and difference gating indices were compared between groups.

\section{Statistical Analyses}

Multiple comparisons were corrected for using the Benjamini-Hochberg procedure, with a false discovery rate of $0.1{ }^{21}$ Within-group and between-group differences were tested using analysis of variance (ANOVA). A one-tailed Pearson correlation was calculated to assess the relationship between PTA (worse ear), HF PTA (worse ear), SNR loss, and gating indices across participants.

\section{Current Density Reconstruction}

Underlying components accounting for the greatest percent variance in CAEP peaks in response to S1 and S2 stimuli were identified using independent component analysis. ${ }^{22}$ Retained components for P50, N1, and P2 S1 and S2 peaks were averaged by participant group in CURRY Scan 7 Neuroimaging Suite (Compumedics Neuroscan). ${ }^{15}$ Grand difference averages were created for each peak by subtraction of S2 from S1 waveforms, and CDR was performed on peak difference averages using standardized low-resolution brain electromagnetic tomography (sLORETA). ${ }^{23}$ Group head models were generated using the boundary element method, ${ }^{24}$ and sLORETA results plotted using an F-statistic distributed color scale on an average magnetic resonance image (MRI; - Fig. 4).

\section{Results}

\section{Audiometry and Speech Perception}

Eleven participants had thresholds at or above $25 \mathrm{~dB}$ HL at two or more frequencies in either ear, and were categorized as the HL group. One NH participant presented with a mild conductive component at $0.250 \mathrm{~Hz}$ in the right ear, but remained in the $\mathrm{NH}$ group as speech perception in noise performance was within normal limits. Two HL participants responded with a threshold above $25 \mathrm{~dB} \mathrm{HL}$ at only one frequency, but were retained in the HL group due to speech perception in noise performance outside of normal limits (mild SNR loss). One individual in the HL group showed significant interaural asymmetry, or a difference of greater than $15 \mathrm{~dB}$ between ears at two or more frequencies. On average, HL participants showed mild-moderate HF HL (-Fig. 1), with significantly worse PTA (worse ear) $(F(1,20)=4.611, p<0.050)$, and HF PTA (worse ear) thresholds than $\mathrm{NH}$ participants ( $F(1$, $20)=45.444, p<0.001)$. No significant difference in SNR loss was found $[F(1,20)=0.823, p=0.375]$.

\section{Auditory Gating in Hearing Loss}

Within-group comparisons of P50, N1, and P2 amplitudes and latencies showed no significant differences between CAEP S1 and S2 waveforms for the $\mathrm{NH}$ or $\mathrm{HL}$ groups ( -Fig. 2A, B). This finding is typically indicative of abnormal inhibitory processes, ${ }^{5}$ but may be due to the mean age (47.546 years) of the $\mathrm{NH}$ group. Age has been reported to negatively impact gating function, ${ }^{25}$ and we have previously observed typical gating function in a young adult cohort (20-22 years) using this paradigm. ${ }^{2,3}$ However, there was a visual trend for amplitude suppression in NH participants for the P2 peak, as well as for a higher amplitude P50 S2 response and similar amplitude response between P2 S1 and S2 peaks in the HL group.

P50, N1, and P2 amplitude ratio and difference indices were calculated to quantify inhibitory function. Only the P2 amplitude difference was significantly decreased in the HL group [F $(1,20)=5.383, p<0.050]$, indicative of inhibitory deficits. The P2 amplitude ratio approached significance for abnormal gating $[F(1,20)=3.811, p=0.065]$. Both results support the hypothesis that central inhibition may be reduced in adults with HL. 
A

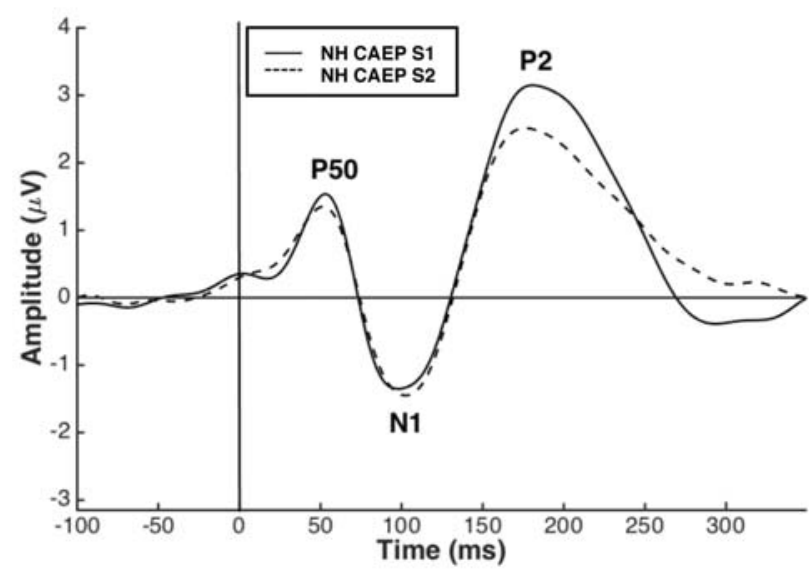

B

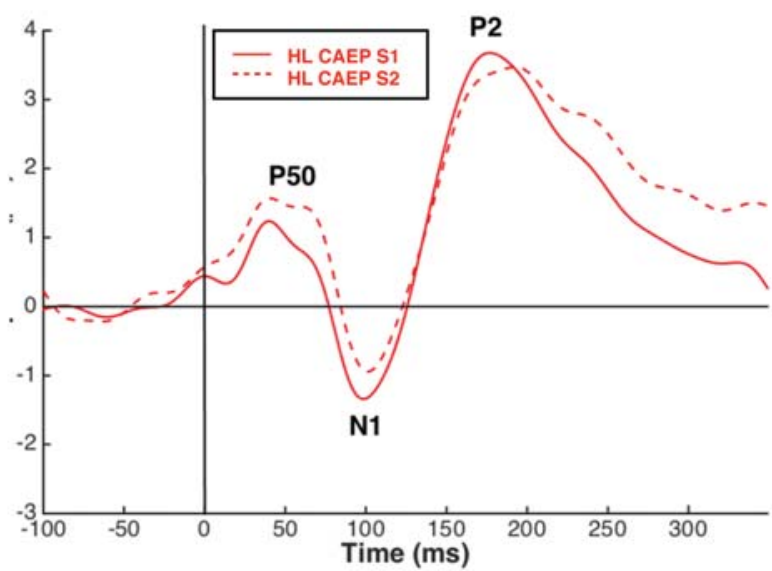

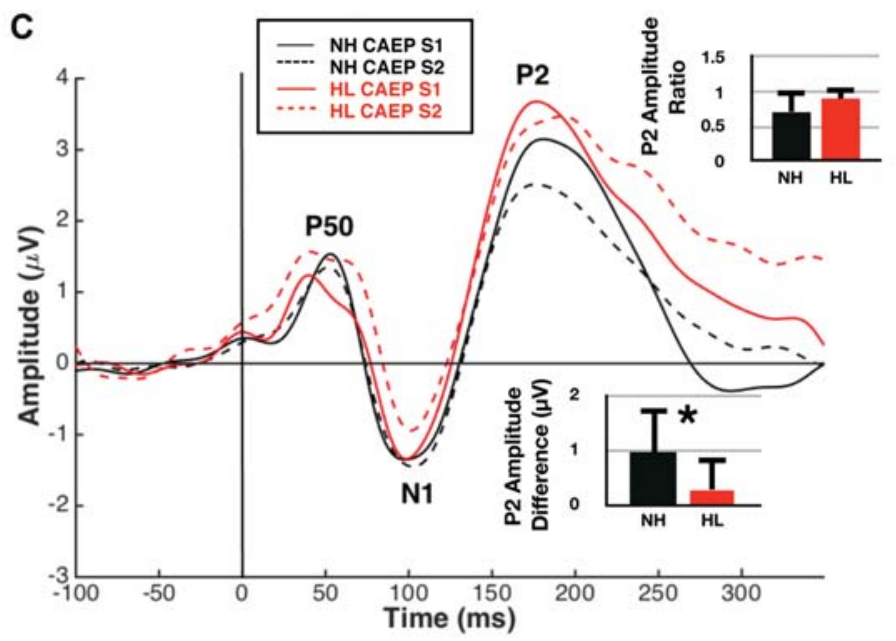

Fig. 2 Auditory gating in normal hearing $(\mathrm{NH})$ and hearing loss $(\mathrm{HL})$. (A) $\mathrm{NH}(n=11)$ CAEP responses to a $0.250-\mathrm{kHz}$ tonal pair. The vertical axis represents amplitude in $\mu \mathrm{V}$ and the horizontal axis milliseconds. (B) $\mathrm{HL}(n=11)$ CAEP responses to a $0.250-\mathrm{kHz}$ tonal pair. (C) NH and HL CAEP comparison. Mean bar graphs illustrate differences in group P2 amplitude gating indices. Error bars illustrate one standard deviation, and one asterisk indicates significance at $p<0.050$. CAEP, Cortical auditory evoked potential.

\section{Auditory Gating Correlations}

A significant negative correlation was observed between PTA and P2 amplitude gating difference $(r=-0.412, p<0.050)$. A similar relationship was found between HF PTA and P2 amplitude gating difference $(r=-0.417, p<0.050$; - Fig. 3A), as well as a significant positive correlation with $\mathrm{P} 2$ amplitude gating ratio $(r=0.379, p<0.050$; - Fig. 3B $)$. These results demonstrate that as auditory thresholds increased, there was an associated decrease in inhibitory processes reflected by the P2 component, consistent with the hypothesis that HL may coincide with deficits in gating function.

\section{Current Density Reconstruction in Auditory Gating}

-Fig. 4 shows CDR results for P50, N1, and P2 difference averages in both groups, with a table listing cortical regions in approximate order from highest activation. Sources of the NH P50 gating component included right superior temporal gyrus (STG) and middle temporal gyrus (MTG). Lateralization of this response was likely due to the use of tonal stimuli. ${ }^{26}$ In addition, right frontal and prefrontal regions such as inferior frontal gyrus (IFG), middle frontal gyrus (MFG), superior frontal gyrus (SFG), and Brodmann area (BA) 6 showed activation. These results are comparable with typical gating function in NH listeners. ${ }^{6,7,27}$ In the HL group, IFG, MFG, and SFG responses were observed, although activation shifted to include mainly temporal regions. Furthermore, an absence of activation in prefrontal cortex was apparent, with left supramarginal and angular gyri responsive instead. A similar lack of prefrontal activation underlying gating deficits has been reported in clinical populations. ${ }^{28}$

Cortical activation for the NH N1 gating component included temporal and frontal regions, as well as precentral gyrus (-Fig. 4), congruent with normal N1 gating function. ${ }^{25} \mathrm{The} \mathrm{HL}$ group showed a shift of activation to temporal areas, as well as lateralization of the temporal response to the left hemisphere. This lateralization during N1 activity is consistent with atypical inhibitory function in elderly participants. ${ }^{25}$

NH P2 gating activity involved the right temporal regions of STG, MTG, and ITG ( - Fig. 4), and left frontal areas (MFG, SFG). In contrast, the HL group presented with only right 

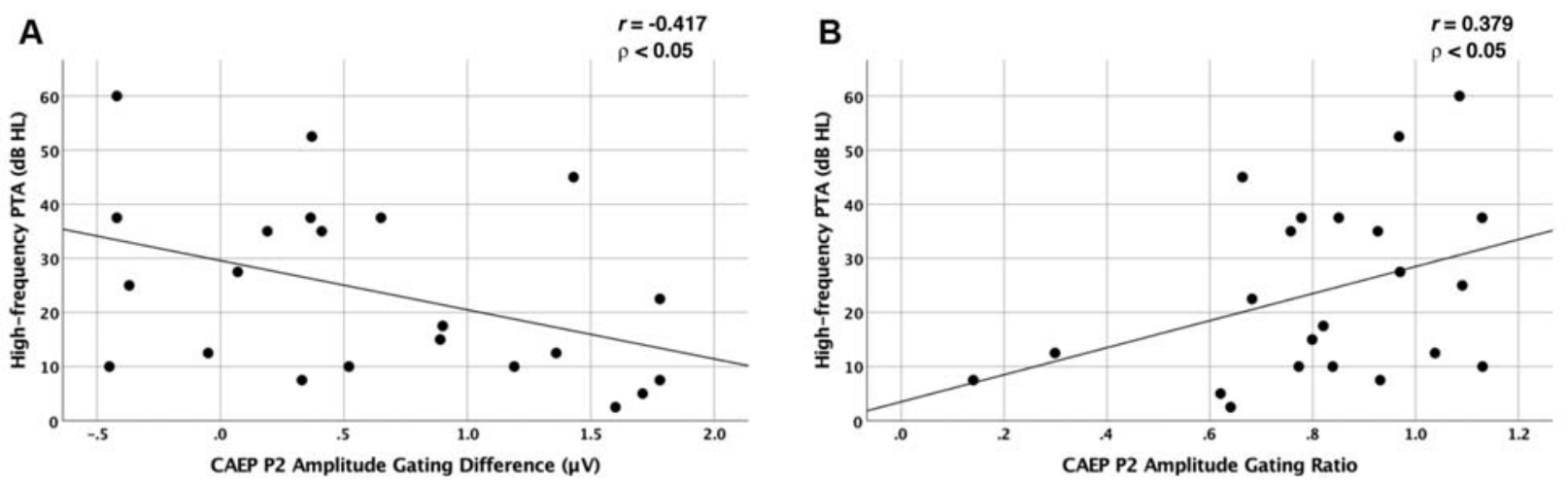

Fig. 3 Threshold correlations with auditory gating. (A) A negative correlation between P2 amplitude gating differences and HF PTA ( $n=22$ ). The Pearson correlation coefficient and level of significance are located in the right corner. P2 amplitude difference values are shown on the horizontal axis in $\mu \mathrm{V}$ and HF PTA on the vertical axis in $\mathrm{dB}$ HL. (B) A positive correlation between P2 amplitude gating ratios and HF PTA. HF, high frequency; HL, hearing loss; PTA, pure-tone average.

A

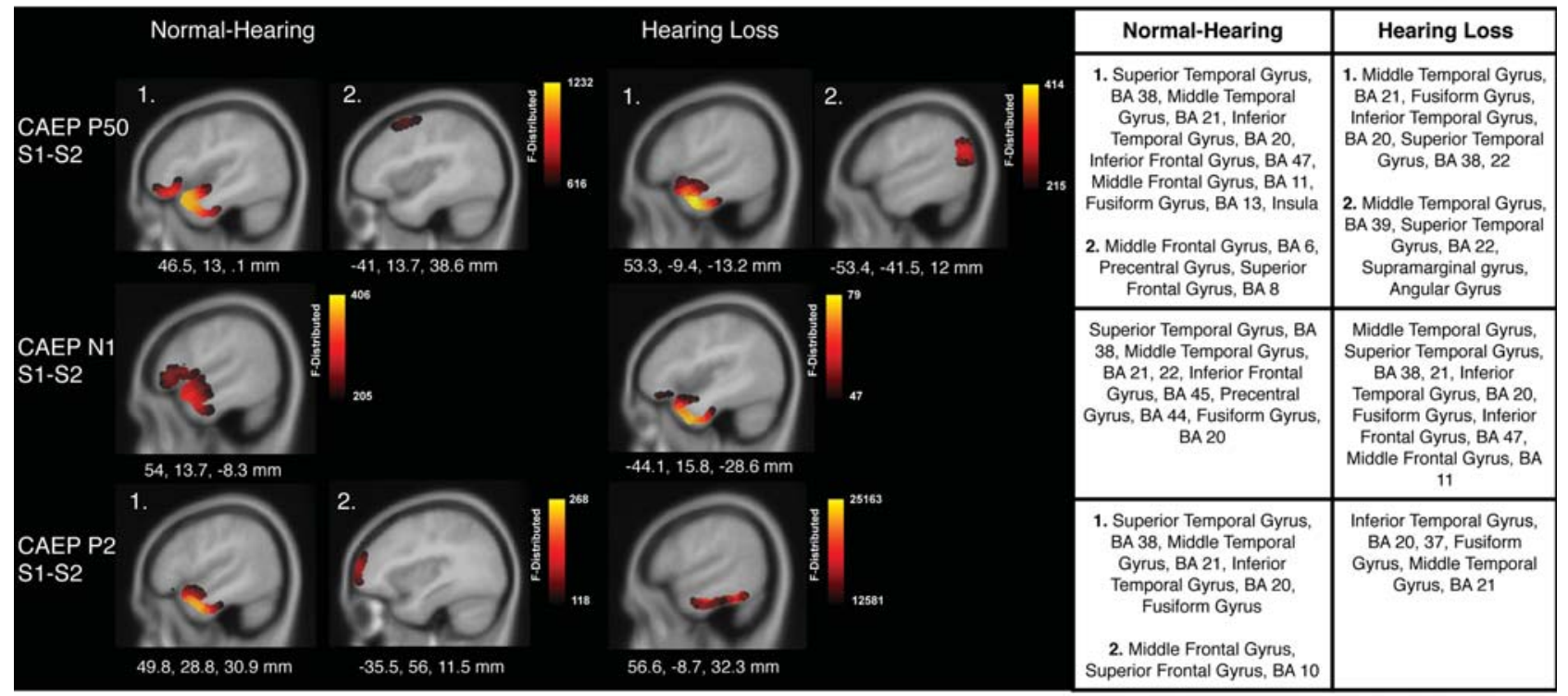

Fig. 4 Auditory gating current density reconstructions. (A) NH and HL CDR for P50, N1, and P2 difference (S1-S2) averages on sagittal MRI slices. The F-distribution scale is located in the right corner, with yellow being the highest level of activation, and MNI (Montreal Neurological Institute) coordinates are listed below each MRI slice. (B) Activated cortical areas, listed in approximate order of highest level of activation. CDR, Current density reconstruction; $\mathrm{HL}$, hearing loss; MRI, magnetic resonance imaging; $\mathrm{NH}$, normal hearing.

temporal activation. A similar reduction in left hemispheric activation underlying the P2 S2 component has been reported in individuals at risk for inhibitory-related deficits. ${ }^{29}$ This result is also consistent with the finding of reduced P2 amplitude gating indices in $\mathrm{HL}(\mathbf{- F i g . ~ 2 C )}$.

\section{Discussion}

We examined auditory gating function in adults with mildmoderate $\mathrm{HL}$ versus $\mathrm{NH}$ controls to determine whether $\mathrm{HL}$ may adversely affect sensory inhibitory mechanisms. Three findings are described: (1) significantly reduced P2 amplitude gating difference indices, consistent with atypical inhibitory function in $\mathrm{HL},(2)$ an association between de- creased P2 gating and increased auditory thresholds, and (3) a possible compensatory temporoparietal gating network in HL.

It should be noted that typical gating function was not observed in the NH group, possibly due to the age of the cohort. In previous studies using the same paradigm, ${ }^{2,3}$ young, $\mathrm{NH}$ adults demonstrated expected gating function consistent with the literature. However, despite an apparent lack of gating in the current $\mathrm{NH}$ group, $\mathrm{P} 50$ gating generators typically present in individuals with normal gating performance were successfully localized. ${ }^{6,7,27}$ This finding suggests that central inhibitory function may have been normal in the NH adults, albeit unobserved via the CAEP measure. Future research should examine the effect of extended age ranges using the described gating 
protocol, particularly as age has been reported to affect gating performance. $^{25}$

While decreased inhibition was identified in $\mathrm{HL}$, it is important to mention that it is unlikely this result is due to reduced audibility. ${ }^{30}$ First, a $0.250-\mathrm{kHz}$ tonal pair was presented at a suprathreshold level $(50 \mathrm{~dB} \mathrm{HL})$ within a frequency region where most subjects demonstrated normal auditory thresholds. Furthermore, a reduction of CAEP amplitude in conjunction with reduced audibility has been reported in the literature, ${ }^{31}$ while we observed either similar or larger CAEP S1 and S2 amplitudes in the HL group versus the $\mathrm{NH}$ group (-Fig. 2C). Rather, this finding is consistent with the hypothesis that there is a corresponding decrement in central inhibition associated with $\mathrm{HL}$, possibly due to a reduction in lateral inhibition resulting from auditory deprivation in adjacent frequency bands. ${ }^{11}$

In addition, we found only the $\mathrm{P} 2$ gating component to specifically reflect inhibitory deficits related to HL. Although the P50 gating component is considered a biomarker of inhibition, ${ }^{5}$ both $\mathrm{N} 1$ and $\mathrm{P} 2$ gating components have been reported to be sensitive to inhibitory deficits. ${ }^{32}$ Furthermore, several CAEP studies have identified the P2 component to be representative of cortical plasticity in HL. P2 amplitude appears to increase in conjunction with elevated thresholds, leading to the hypothesis that larger amplitude may arise from decreased inhibition associated with HL and subsequent "effortful listening." Thus, the P2 gating component is likely representative of plasticity in cortical inhibitory function related to acquired HL.

In support of this hypothesis, CDR results revealed a qualitative difference between inhibitory networks in $\mathrm{NH}$ and HL adults (-Fig. 4). NH gating function was localized in temporal, frontal, and prefrontal cortical regions, including activation of BA 6 , consistent with previous gating studies. $^{6,7,27}$ In contrast, HL gating function was decreased in frontal and prefrontal regions, with a shift to temporal and parietal areas. Similar gating activity in parietal regions was observed in typical subjects, ${ }^{6,27}$ suggesting that those with HL may use an incomplete or secondary gating network in comparison with $\mathrm{NH}$ adults. Along these lines, there was a reduction in frontal activation underlying the HL P2 gating response. This specific finding could be a result of resource reallocation in HL. For instance, Campbell and Sharma ${ }^{15}$ showed that $\mathrm{HL}$ adults reallocate passive processing of auditory input from temporal to frontal cortex, indicative of recruitment of cognitive resources for the processing of degraded lower order auditory information. Thus, in HL, frontal cortical resources required for typical gating function may not be available, leading to the development of a compensatory temporoparietal gating network.

\section{Study Limitations}

While HL appeared to be related to decreased inhibition in the present study, it will be important in future research to tightly control for and examine the effects of specific types of $\mathrm{HL}$, including configuration, laterality, and severity, on the CAEP amplitude gating response. For instance, the participants included in the HL group demonstrated a range of hearing thresholds across varying frequencies, which may have pre- cluded findings of atypical inhibition specific to patterns of HL. Along these lines, it will also be necessary to better understand possible age effects and interactions with HL on CAEP amplitude gating indices. The age range included in this study was broad, and may have acted as a confounding variable in observing a typical gating response for the $\mathrm{NH}$ group. However, post-hoc analyses showed no significant correlation between CAEP amplitude gating indices and age across groups, suggesting that group differences in gating function were indeed due to $\mathrm{HL}$ rather than age effects. In any case, additional research is required to better understand and appropriately implement auditory gating as a clinical measure for central disorders such as tinnitus, ${ }^{2,3}$ especially in the presence of HL.

\section{Conclusion}

This study examined the effects of mild-moderate sensorineural HL on auditory gating, an inhibitory measure used in clinical populations, to better understand the impact of reduced auditory input on sensory inhibitory mechanisms. Using CAEPs recorded via high-density EEG in response to tonal pairs, we found decreased inhibition in HL reflected by P2 amplitude gating indices, which correlated with auditory thresholds. In $\mathrm{NH}$ adults, the gating response was localized in temporal, frontal, and prefrontal cortices, while adults with HL showed mainly temporal and parietal responses. Overall, our results suggest decreased inhibition underlying auditory gating in HL. These outcomes should be considered when implementing auditory gating as a clinical measure, especially in disorders such as tinnitus.

\section{Funding}

This work was supported by the Hearing Health Foundation Emerging Research Grant, through support from Les Paul Foundation (2016), and the Texas Speech-LanguageHearing Foundation Lear Ashmore Research Fund (2016).

\section{Conflict of Interest}

None declared.

\section{References}

1 Hamilton HK, Williams TJ, Ventura J, et al. Clinical and cognitive significance of auditory sensory processing deficits in schizophrenia. Am J Psychiatry 2018;175(03):275-283

2 Campbell J, Bean C, LaBrec A. Normal hearing young adults with mild tinnitus: reduced inhibition as measured through sensory gating. Audiology Res 2018;8(02):214

3 Campbell J, LaBrec A, Bean C, Nielsen M, So W. Auditory gating and extended high-frequency thresholds in normal-hearing adults with minimal tinnitus. Am J Audiol 2019;28(1S(:209-224

4 Chien YL, Hsieh MH, Gau SS. P50-N100-P200 sensory gating deficits in adolescents and young adults with autism spectrum disorders. Prog Neuropsychopharmacol Biol Psychiatry 2019; 95:109683

5 Javitt DC, Freedman R. Sensory processing dysfunction in the personal experience and neuronal machinery of schizophrenia. Am J Psychiatry 2015;172(01):17-31

6 Knott V, Millar A, Fisher D. Sensory gating and source analysis of the auditory P50 in low and high suppressors. Neuroimage 2009; 44(03):992-1000 
7 Knott VJ, Fisher DJ, Millar AM. Differential effects of nicotine on P50 amplitude, its gating, and their neural sources in low and high suppressors. Neuroscience 2010;170(03):816-826

8 Vlcek P, Bob P, Raboch J. Sensory disturbances, inhibitory deficits, and the P50 wave in schizophrenia. Neuropsychiatr Dis Treat 2014;10:1309-1315

9 Fuerst DR, Gallinat J, Boutros NN. Range of sensory gating values and test-retest reliability in normal subjects. Psychophysiology 2007;44(04):620-626

10 Noreña AJ, Farley BJ. Tinnitus-related neural activity: theories of generation, propagation, and centralization. Hear Res 2013; 295:161-171

11 Eggermont JJ. Acquired hearing loss and brain plasticity. Hear Res 2017;343:176-190

12 Recanzone G. The effects of aging on auditory cortical function. Hear Res 2018;366:99-105

13 Harkrider AW, Plyler PN, Hedrick MS. Effects of age and spectral shaping on perception and neural representation of stop consonant stimuli. Clin Neurophysiol 2005;116(09):2153-2164

14 Bertoli S, Probst R, Bodmer D. Late auditory evoked potentials in elderly long-term hearing-aid users with unilateral or bilateral fittings. Hear Res 2011;280(1-2):58-69

15 Campbell J, Sharma A. Compensatory changes in cortical resource allocation in adults with hearing loss. Front Syst Neurosci 2013;7:71

16 Bertoli S, Bodmer D. Novel sounds as a psychophysiological measure of listening effort in older listeners with and without hearing loss. Clin Neurophysiol 2014;125(05):1030-1041

17 Parry LV, Maslin MRD, Schaette R, Moore DR, Munro KJ. Increased auditory cortex neural response amplitude in adults with chronic unilateral conductive hearing impairment. Hear Res 2019;372:10-16

18 Smith DM, Grant B, Fisher DJ, Borracci G, Labelle A, Knott VJ. Auditory verbal hallucinations in schizophrenia correlate with P50 gating. Clin Neurophysiol 2013;124(07):1329-1335

19 Newman CW, Jacobson GP, Spitzer JB. Development of the Tinnitus Handicap Inventory. Arch Otolaryngol Head Neck Surg 1996; 122(02):143-148

20 Delorme A, Makeig S. EEGLAB: an open source toolbox for analysis of single-trial EEG dynamics including independent component analysis. J Neurosci Methods 2004;134(01):9-21
21 Benjamini Y, Hochberg Y. Controlling the false discovery rate: a practical and powerful approach to multiple testing. J R Stat Soc Series B Stat Methodol 1995;57:289-300

22 Makeig S, Jung TP, Bell AJ, Ghahremani D, Sejnowski TJ. Blind separation of auditory event-related brain responses into independent components. Proc Natl Acad Sci U S A 1997;94(20): 10979-10984

23 Pascual-Marqui RD. Standardized low-resolution brain electromagnetic tomography (sLORETA): technical details. Methods Find Exp Clin Pharmacol 2002;24(Suppl D):5-12

24 Fuchs M, Kastner J, Wagner M, Hawes S, Ebersole JS. A standardized boundary element method volume conductor model. Clin Neurophysiol 2002;113(05):702-712

25 Cheng CH, Baillet S, Lin YY. Region-specific reduction of auditory sensory gating in older adults. Brain Cogn 2015;101:64-72

26 Lamminmäki S, Parkkonen L, Hari R. Human neuromagnetic steady-state responses to amplitude-modulated tones, speech, and music. Ear Hear 2014;35(04):461-467

27 Mayer AR, Hanlon FM, Franco AR, et al. The neural networks underlying auditory sensory gating. Neuroimage 2009;44(01): $182-189$

28 Josef Golubic S, Aine CJ, Stephen JM, Adair JC, Knoefel JE, Supek S. MEG biomarker of Alzheimer's disease: absence of a prefrontal generator during auditory sensory gating. Hum Brain Mapp 2017; 38(10):5180-5194

29 Rihs TA, Tomescu MI, Britz J, et al. Altered auditory processing in frontal and left temporal cortex in 22q11.2 deletion syndrome: a group at high genetic risk for schizophrenia. Psychiatry Res 2013; 212(02):141-149

30 McClannahan KS, Backer KC, Tremblay KL. Auditory evoked responses in older adults with normal hearing, untreated, and treated age-related hearing loss. Ear Hear 2019;40(05): 1106-1116

31 Lightfoot G. Summary of the N1-P2 cortical auditory evoked potential to estimate the auditory threshold in adults. Semin Hear 2016;37(01):1-8

32 Lijffijt M, Moeller FG, Boutros NN, et al. Diminished P50, N100 and P200 auditory sensory gating in bipolar I disorder. Psychiatry Res 2009;167(03):191-201 